\title{
DISABILITY AND MENTAL ILL-HEALTH-IMPLICATIONS FOR SOCIAL POLICY AND RECOMMENDATIONS IN NIGERIA
}

\section{Adeoye O. Oyewole}

Department of Psychiatry, Ladoke Akintola University of Technology, Ogbomosho, Oyo

Email: adeoyewole2000@yahoo.com

Cite this article:

Adeoye O. Oyewole (2022), Disability and Mental IllHealth-Implications for Social Policy and Recommendations in Nigeria. African Journal of Social Sciences and Humanities Research 5(1), 110. DOI: $10.52589 / A J S S H R-$ 00STOL2V.

\section{Manuscript History}

Received: 19 Dec 2021

Accepted: 12 Jan 2021

Published: 22 Jan 2022

Copyright $\odot 2020$ The Author(s). This is an Open Access article distributed under the terms of Creative Commons AttributionNonCommercial-NoDerivatives 4.0 International (CC BY-NC-ND 4.0 ), which permits anyone to share, use, reproduce and redistribute in any medium, provided the original author and source are credited.
ABSTRACT: The conceptual definition of disability has been predicated on the quest to balance the medical paradigm with the equally relevant social paradigm of disability. In this paper, I review current models of disability and their impact on interrelationships between disability and mental health disorders within the context of African socio-cultural concepts. I also propose a mental health paradigm through the biopsychosocial model in the management of the complex issues associated with disability. Health workers collaborating with religious and sociocultural institutions can identify those at risk of grave mental health consequences and offer support to the individual and the family through proper counselling and guidance.

KEYWORDS: Biopsychosocial, counselling, disability, health, Africa 


\section{INTRODUCTION}

Disability is a multi-dimensional concept and an experience arising from the interaction of health conditions and the environment (Organization, 2001). Over the years, the conceptual definition of disability has been predicated on the quest to balance the medical paradigm with the equally relevant social paradigm of disability. Current global estimates indicate that around $10 \%$ of people live with a disability and this number is growing as a result of some factors including increased survival rates for children with disability and increased population life expectancies (Mont, 2007; Natalie, 2011; Perrin, 2002; Sawyer et al., 2007). For instance, it is estimated that over 15 percent of Nigerians are living with disability excluding those with intellectual and sensory impairments. In Africa, the community of persons with bodily disabilities is growing at a geometric rate since strong factors of predisposition such as wars, famine, terrorism, road and domestic accidents, medical misdiagnosis, poor compliance with immunization against polio and other killer diseases, collapsed buildings, poor obstetric care, rising prevalence of non-communicable diseases and many others are common.

In a survey of 1093 respondents conducted in Kogi and Niger states of Nigeria in 2005, the most common disabilities involved vision (37\%), mobility (32\%) and hearing (15\%). They were majorly beggars since over half of them were less than 21 years and had no money (Natalie, 2011). In Africa, the majority of persons with disabilities almost always have a raw deal in terms of acceptance, access and respect for their rights. Our society has not come to terms with the fact that there is ability in disability. Persons living with disabilities are hardly given the necessary social support as they are cursed, insulted and at times physically and emotionally abused for bringing bad luck to the family (Jide, 2012). Disability, therefore, is not just a medical problem. It is a complex phenomenon reflecting the interaction between features of a person's body or mind and the dynamics of the society in which he or she lives.

This paper attempts, therefore, to clarify conceptual challenges in defining disability by throwing up the several models that exist and their historical implications to care, the precarious interrelationships between disability and mental health disorders, the peculiarity of the African sociocultural concept concerning disability, elucidation of the mental health paradigm through the biopsychosocial model and proffering workable interventions in the management of the complex issues associated with disability.

\section{Conceptual Issues in Defining Disability}

The question of the definition of 'person with a disability and how persons with disabilities perceive themselves are knotty and complex. It is no accident that these questions are emerging at the same time that the status of persons with disabilities in society is changing dramatically (Deborah, 2010). The definition of disabilities has had a very close relationship with the quality of policy response it generates just as there is a historical background to the definition of disabilities which is precariously linked to the quality of attention it has elicited from both government and society from time to time.

The moral model is historically the oldest and is less prevalent today although may still exist in some subcultures in Africa. Same African cultural practices may associate disability with sin and shame complicated by feelings of guilt, even if such feelings are not overtly based on religious doctrines. For the individual with a disability, this model is particularly burdensome. On many occasions, families have had to hide away disabled family members, keeping them 
out of school and excluded from any chance of having any meaningful role in society. This model generated much social ostracism and self-hatred. This model has little or no consideration for the mental health needs of those with disabilities.

The medical model views disability as a result of a physical condition intrinsic to the individual which may reduce the individual quality of life and cause clear disadvantages to the individual. This model tends to believe that caring or at least managing disabilities mostly revolves around identifying the disability from an in-depth clinical perspective, understanding it, and learning to control or alter its course.

In this model, the individual with a disability is in the sick role who is expected to be excused from the normal obligations of the society like going to school, getting a job, taking on family responsibilities and are expected to come under the authority of the medical profession to get better. Until recently, most disability policy issues have been regarded as health issues while physicians are seen as the primary authorities in this policy era. This paradigm was so strong that it impacted the social security system where disability is defined as the inability to work which is consistent with the role of the person with a disability as sick, however, with very little consideration for their psychological issues.

The rehabilitation model is quite similar to the medical model where the person with a disability is regarded as in need of services from a rehabilitation professional who can provide training, therapy, counselling or other services to make up for the deficiency caused by the disability. Historically, this model gained acceptance after World War II when many disabled veterans needed to be reintroduced into society (David, 2003; Susan, 1996).

However, there are strong points against the medical and rehabilitation models. Many disabilities and chronic medical conditions may never be cured just as persons with disabilities are quite capable of participating in societal activities such that the practices of confinement and institutionalization that emanate from the concept of the sick role may not be acceptable.

The disability or social model is a product of the activities of the disability right and the independent living movements. This model views disability as a normal aspect of life, not as deviance and rejects the notion that persons with disabilities are in some inherent way defective. There is a consideration of the fact that most people will experience some form of disability either permanent or temporary throughout their lives which may affect the way we design our environment or our systems in order not to discriminate against the disabled. This model tackles the cultural habit of regarding the condition of the person rather than the built environment or the social organization of activities as the source of the problem. This model appears to be most sensitive to the psychological needs and social challenges of persons with disabilities. These various models can be viewed in the context of their impact on the mental wellbeing of those with disabilities as arising from their core emphasis.

\section{Interrelationships between Disability and Mental health disorders}

People with disabilities appear to be at greater risk of mental health problems than the general population and, therefore, makes a disproportionate contribution to mental health internationally. Global estimates indicate that about $10 \%$ of people live with a disability (Mont, 2007). Mental disorders account for $20 \%$ of the total burden of disease worldwide while depression alone is the number one contributor to the non-fatal burden of disease and disability for both high and low/middle-income countries (Lopez et al., 2006). For individuals with 
disabilities, mental health complications increase the burden and may invariably jeopardize the ultimate objective of the social model of disability.

Most evidence-based research done on the association between disability and mental health is cross-sectional limiting the conclusion that could be drawn about causality. However, about three hypotheses explain how people with disabilities have poorer mental health than their nondisabled peers. First, the experience of living with a disability or having a health condition or impairment associated with a disability could lead to mental health problems. Secondly, people with mental health problems could be more likely to subsequently become disabled and lastly, other factors such as the socio-economic and socio-cultural factors might independently increase the risk of disability to mental ill-health (Honey et al., 2010).

\section{Disability Increases the Risk of Developing Mental Disorder}

There is ample evidence that the experience of any form of disability can cause mental disorders. Some disease processes can affect the brain directly and also some of the medications used can have negative mental health effects (Malek-Ahmadi \&Hilsabeck, 2007). Chronic disability is also capable of inflicting psychological burdens through trauma, declining health, unpleasant treatments, stigma, loss of social support and relationship breakdown. Those who have intellectual disabilities are characterized by poor problem-solving abilities, poor emotional control, communication difficulties and a high rate of physical and sexual abuse may also increase vulnerability to mental health problems (Dagna \& Lindsay, n.d.; Prince et al., 2007). Disabling physical illnesses like myocardial infarction, stroke, HIV infection and injury have strong associations with mental illness. Confinement to a bed/chair, spinal pain, neurologic and gastrointestinal diseases are strongly associated with increased risk of depression (Bruce \& Hoff, 1994). However, some considerable individual variability exists in how an individual's mental health may be affected by disability onset.

Some folks have scores that show no worsening in their mental health consisting of about $65 \%$ while a smaller group, about $16 \%$ of the population, may experience rapid mental health deterioration after disability onset. They are more likely to be younger, unemployed, uneducated and untrained before the onset of disability, and are usually from dysfunctional families with poor uneducated parents (Kariuki et al., 2011).

\section{Mental Disorder as Risk for Disability.}

The physical health of people with mental disorders is notoriously poor (Yeomans \& Citrome, 2005). Disorders like heart disease, diabetes, stroke, HIV/AIDS, tuberculosis and accidental/non-accidental injuries are strongly associated with mental illness through lifestyle risk factors such as obesity, smoking, low adherence to condom use, complications of the antipsychotic drugs that may cause heart diseases movement disorders, osteoporosis and seizures (Commission., 2006).

When mental disorders are associated with a pre-existing illness or impairment, there is a worse outcome through poor functioning. Several factors may contribute to such as being less likely to seek treatment for early symptoms due to cognitive impairment, social isolation, distrust of medical staff and lack of social skills. They may also be less likely to receive a timely diagnosis due to difficulty in accurately conveying symptoms, the reduced rate at which folks with mental disorders receive evidenced-based checks and diagnostic tests; also there is the problem of diagnostic overshadowing where all reported symptoms are seen as related to the mental 
disorder. There is evidence to show that mentally ill folks do not receive the same level of treatment as others through social discrimination. Lastly, people with mental disorders are at greater risk by non-adherence to the medical and behavioural treatment regimen.

\section{Convergence Risk Factor}

Mental disorders may share common risk factors with other disabilities. They often experience social disadvantage, low socioeconomic status and inadequate social support. There is also strong evidence linking the same factors to worse mental health. Downward social mobility has been attributed to both disability and mental disorders, largely due to exclusion from the labour market and the costs associated with a disability which are often secondary to stigma and discrimination that exacerbates the impact of disability through social and economic factors (Emerson et al., 2009; Emerson \& Hatton, 2007).

\section{The Subclinical Psychological Disorders.}

However, some behavioural manifestations could be subclinical especially among those experiencing a new form of disability. They will have to cope with life transitions, value changes and socio-economic challenges across their life span. From a sociological perspective, people who experience disability for the first time also have to deal with the role of family, cross-cultural issues, adjustments, the consequences of negative demeanour towards people with disabilities as a whole. Their system of life and living has changed in many different ways, meaning they must endure a process of adjustment and self-evaluation. The experience of a new disability is similar to a mourning process (and might be equated to the loss of a loved one) which includes shock, denial, anger/depression and adjustment/acceptance. People progress through these stages at their own pace. Failure to achieve acceptance may result in developing mental disorders when the individual cannot come to terms with the disability. Acceptance does not necessarily mean the person is happy about the disability but active relinquishment of any false hopes as well as successful adaptation of new roles based on realistic potentials and limitations. The person might benefit from interaction with others who have similar experiences (Wendy, 2012).

However, there is no clear study that has unequivocally demonstrated the incidence of psychiatric disorders in a cohort of disabled persons since conceptually, there is a blurring of definition between disability the way it is defined here and mental illness which is regarded as a form of disability. This opens an avenue for future research using this concept of definition to demonstrate the actual prevalence of psychiatric disorders in this cohort.

\section{African sociocultural perspectives and Disability}

There is a paucity of literature investigating the association of mental illness with disability in Africa but there is an apparent growth in the population of individuals with disability possibly due to the apparent preponderance of factors of predisposition to disability in Africa as mentioned earlier. The complicating socio-cultural forces are equally very potent in Africa rooted in myths, superstitions and taboos directed against persons with disabilities. The sick role that conceptualizes disability in Africa is quite similar to that for mental illness and possibly fixated on the most primitive definition of disability as a consequence of sin as stated in the moral model definition. Africans are animists, an orientation that still permeates most of the foreign adopted religious practices like Christianity. Disability, just like mental illness may be viewed as being caused by supernatural forces that must be appeased. This belief concept 
interferes with the mental well-being of persons with disabilities, predisposes them to develop mental illness through social discrimination and social ostracism that frustrates quality care and support. The tendency to be stigmatized is heightened which frustrates the fundamental philosophy of the social model and the underlying principles of the World Health Organization's and United Nations' definition of disability.

Several scholars have pointed out that an individual's beliefs about disability form the template upon which attitudes towards people with disabilities are formed (Fishbien \& Ajzen, 1975; Madu \& Yahaya, 2014; Ozoji, 1991). These attitudes invariably determine how folks with disabilities are treated without supportive societal standards to the needs and aspirations of people with disabilities (Hahn, 1986).

People with mental illness are killed as part of rituals, practices that flow from various beliefs that people hold about disability. Many who hold negative beliefs about persons with mental illness claim that their hands are unclean and have violated the tradition of the communities. In other cases, a mentally ill person is simply labelled as a witch and subsequently burnt to death (Oko, 2003; Omiegbe, 1998, 2001). For instance, folks with occulo-cutaneous albinism are sometimes isolated, trafficked and even killed and as a result, people with albinism live in hiding. The killing of people with albinism for rituals is fueled by the belief that their body parts could be used for portions that will make one wealthy or prolong one's life (Anumihe, 2008; Oko, 2003; Okoro, 1975). There are vivid accounts of how some of these folks are kidnapped and subsequently used for ritual purposes. In one report presented by Odejobi; individuals who were fortunate to escape from their kidnappers' recounted stories of how people were kidnapped and killed for ritual purposes. Such ritual killings have either a personal or a communal dimension (McVeigh, 2007; Odejobi, 2010).

These scenarios illustrate unequivocally, the challenges that folks with disabilities face in a developing country like Nigeria which is not just merely attitudinal but deeply rooted in our culture. Since culture is like the software that drives human behaviour and determines largely our attitudes; there is a need for a paradigm that can wholesomely interrogate the sociocultural dimensions as touching the care of the disabled people.

Also, Parents or guardians of children with disabilities send their children out for alms-begging since the sight of such children is expected quite likely to evoke a sense of sympathy from members of the society, especially from those that take alms-giving as an obligation and those who refuse to beg are usually threatened with beating or refused food. There are cases where the punishment for refusal is more severe, for example, chasing the child with disabilities away from home (Odejobi, 2010).

For low-income countries like Nigeria, mental health and disability are often given the lowest health priority by the authorities and this goes a long way to deepen the stigma and discrimination faced by this population. This group of persons are challenged twice; on one hand, they struggle with their disabilities and on the other; they are stereotyped and prejudiced due to misconceptions about mental illness and disability (Omiegbe, 1995).

These reports highlight the role of culture in discriminatory practices against persons with disabilities, which seems consistent with Oliver's variant of the social model of disability. In his seminal work, Oliver seeks to provide conclusive evidence that disability 'as a category can only be understood within a framework which suggests that it is culturally produced and 
socially structured'(Oliver, 1990). The point is that the discriminatory practices against persons with disabilities are sustained by culture and in doing so reinforce the dominant perspectives regarding disability. Therefore, it is not only the case that people with disabilities are killed based on disability beliefs (e.g., superstition), but these killings are also ritualized; they are ritualized because they arise from a particular culture embedded in a particular worldview. The killings of people with disabilities in Nigeria can be considered aspects of cultural practices or culture where perpetrators pick out such persons and kill them for ritual purposes in pursuit of success and wealth. This is the point that we made above about the communal dimension of ritual killings and is in line with Abang's remark that people with disabilities are targeted for their social and economic benefits (Abang, 1988).

Religion as a subset of culture has also been noted to have a very potent impact just as Olupona noted that various ritual practices carried out in many communities across Nigeria are grounded in some form of African traditional religion (ATR) (Olupona, 1991). This claim is important considering that disability beliefs and the attitudes towards people with disabilities are not vehemently sanctioned by the two dominant religions in Nigeria which raises certain questions about the deep connection and attachment to practices and activities that are related to ATR which Oyebode reported that the influence exerted by ATR can be seen in the attitudes of Nigerians towards oaths administered via the Holy Bible and the Holy Koran compared to that of the ATR. This observation illustrates the point that many Nigerians that are still wedded to the ATR are goaded by beliefs embedded in this religion to engage in ritual killings of persons with disabilities (Oyebode, 2009).

\section{Mental health paradigm to the Issues of Disability}

In 1980, the World Health Organization (WHO) introduced a framework for working with disabilities, publishing the international classification of impairments, disabilities and handicaps. The proposed framework approached disability using the terms impairment, handicap and disability. Impairment is defined as a loss or abnormality of physical bodily function, of the logic-psychic origin or physiological or anatomical origin while disability is viewed as any limitation or function loss deriving from an impairment that prevents the performance of an activity in the time-lapse considered normal for a human being while handicap is defined as the disadvantaged condition deriving from impairment or disability limiting a person performing a role considered normal in respect of their age, sex and sociocultural factors(WHO, 2004).

These technical definitions have strong correlations with a holistic view of health which is in consonance which the World Health Organization defines as not only the absence of infirmity but a state of physical, emotional and spiritual wellbeing. The disability model had expanded the scope of defining disability from the parochial, narrow definition of the medical model to accommodate all other factors crucial for the overall wellbeing of such persons. The international classification of functioning, disability and health (ICF) defines disability as an umbrella term for impairments, activity limitations and participation restrictions. Disability, therefore, is seen as the interaction between individuals with health conditions like cerebral palsy, Down syndrome or depression and environmental factors which could be negative attitudes, inaccessible transportation and limited social supports.

The altered language and words used show marked changes in emphasis from talking in terms of disease or impairment to talking in terms of levels of health and functioning. This change is 
consistent with the widespread acceptance of the social model of disability. These redefinitions are strongly correlated with the concept of mental wellbeing beyond the physiological or structural loss of function. Mental wellbeing is defined by the World Health Organization as a state of well-being in which every individual realizes his or her potential, can cope with normal stresses of life, can work productively and fruitfully and can make a contribution to his or her community (Engel, 1980).

The Bio-psycho-social model proposed by Engel views mental wellbeing as a holistic concept that considers man not only from the biological perspective alone but also takes consideration of the psychological and social dimensions which is closely related to the disability or social model (American Psychiatric Association, 1994). The behaviour and psychological issues adopted in this context may be used interchangeably here to describe a set of conditions characterized by changes in thinking, mood or behaviour that are associated with distress that are personal and experienced by significant others with impaired social functioning. However, certain discrete mental disorders are adopted to describe a set of symptoms that are clinically diagnosable under the Diagnostic and Statistical Manual of mental disorders volume IV-TR (American Psychiatric Association, 2013). This model provides a template for interrogating the varied issues of disability that the models have attempted to do individually.

\section{RECOMMENDATIONS}

The mental health paradigm of interrogating issues of disability is fully captured in the biopsycho-social model proposed by Engel as a holistic template of intervention. It requires a multi-disciplinarian approach for practical outworking. The challenge for social policy planners is the capacity to identify and confront the local social construct of disability rooted in the culture that feeds the sick role which perpetuates the stigmatization of mental illness and disability. The biopsychosocial model sufficiently engages the issues pragmatically raised with disability either in planning for varied shades of intervention, challenging the various models of care that have existed in the care and planning for the disabled. This template does not just focus on the functional disability alone but is competent in addressing various psychological and psychiatric problems that may arise as a result of the disability and stigmatization. This proposed model will address the social, psychological and biological issues in planning and advocacy for the rights of the disabled. The social workers in collaborating with the religious and socio-cultural institutions can identify those at risk of grave mental health consequences and offer support to the individual and the family through proper counselling and guidance. The rehabilitative effort inclusive of mental health consultation can be effectively coordinated through this multidisciplinary approach especially in a low-income country like Nigeria. 


\section{REFERENCES}

Abang, T. B. (1988). Disablement, disability and the Nigerian society. Disability, Handicap \& Society, 3(1), 71-77.

American Psychiatric Association. (1994). Diagnostic and Statistical Manual of Mental Disorders (4th ed.). American Psychiatric Association.

American Psychiatric Association. (2013). American Psychiatric Association: Diagnostic and Statistical Manual of Mental Disorders (5th ed.).

Anumihe, I. (2008, October 11). Fear of ritualists: Albinos go underground. Sun Newspaper.

Bruce, M. L., \& Hoff, R. A. (1994). Social and physical health risk factors for first-onset major depressive disorder in a community sample. Social Psychiatry and Psychiatric, Epidemiology. 29(4), 165-171.

Commission., D. R. (2006). Equal Treatment: Closing the Gap a Formal Investigation into Physical Health Inequalities Experienced by People with Learning Disabilities and/or Mental Health Problems.

Dagna, D., \& Lindsay, W. (n.d.). People with mental health problems. Clinical Psychology and People with Intellectual Disabilities. (E. Emerson, C. Hatton, J. Bromley, A. Caine, R. Gone, \& K. Dickson (eds.); 2nd ed.). Wiley.

David, P. (2003). Disability studies and the Disability perspective. Disability Studies Quarterly, 23(1), 142-148.

Deborah, K. (2010). The Definition of Disability. The Center for an Accessible Society. 510, 251-4347.

Emerson, E., \& Hatton, C. (2007). The Contribution of Socio-Economic Position to the Health Inequalities Faced by Children and Adolescents with Intellectual Disabilities in Britain. American Journal of Mental Retardation, 112, 140-150.

Emerson, E., Madde, R., Robertson, J., Graham, H., Hatton, C., \& Llewellyn, G. (2009). Intellectual and Physical Disability, Social Mobility, Social Inclusion and Health. Centre for Disability Research.

Engel, G. L. (1980). The clinical application of the Biopsychosocial Model.American Journal of Psycology.1377 (5), 535-544.

Fishbien, M., \& Ajzen, T. (1975). Belief, attitudes, and intentions and behaviour: An introduction to theory and research, Addison Wesley Publishing Company, Manila.

Hahn, H. (1986). Public support for rehabilitation in programs: The analysis of US disability policy. Disability, Handicap \& Society, 1(2), 121--138.

Honey, A., Emerson, E., Llewellyn, G., \& Kariuki, M. (2010). Mental Health and Disability. In S. JH \& B. M (Eds.), International Encyclopedia of Rehabilitation.

Jide, O. (2012). The plight of Persons with Disabilities in Nigeria.PunchNewspapers.

Kariuki, M., Honey, A., Emerson, E., \& Llewellyn, G. (2011). Mental Health Trajectories of Young People after Disability Onset. Disability Health Journal, 4(2), 91-101.

Lopez, A. D., Mathers, C. D., Ezzati, M., Jamison, D. T., \& Murray, C. J. L. (2006). Global burden of disease and risk factors.

Madu, F., \& Yahaya, I. (2014, October 8). Deaf persons raise alarm over the future. This Day.

Malek-Ahmadi, P., \& Hilsabeck, R. C. (2007). Neuropsychiatric Complications of Interferons: Classification, Neurochemical Bases, and Management. Annals of Clinical Psychiatry, 19(2), 113-123.

McVeigh, T. (2007, December 9). 'Children abused, killed as witches in Nigeria'’. Guardian. 
Mont, D. (2007). Disability in Australia: Trends in Prevalence, Education, Employment and Community Living. Canberra: AIHW. Australian Institute of Health and Welfare.

Natalie, S. (2011). The Face of disability in Nigeria: A Disability Survey in Kogi and Niger States.DCID; 1, 22, 11.

Odejobi, T. (2010, February 7). The worth of the Nigerian life. Tribune.

Oko, E. O. (2003). Extra-judicial killings in Nigeria: The case of Afikpo town- a paper presented at the 17th International Conference of the International Society for the Reform of Criminal Law, 24-28 August, The Hague, Netherlands,

Okoro, A. N. (1975). Albinism in Nigeria: A clinical and social study. British Journal of Dermatology, 92(5), 485-492.

Oliver, M. (1990). The politics of disablement, MacMillan.

Olupona, J. K. (1991). Kingship, religion, and rituals in a Nigerian community, Almqvist \& Wiksell International, Stockholm.

Omiegbe, O. (1995). The Handicapped and begging-A paper presented at the Fifth NationalConference at the Department of Special Education.

Omiegbe, O. (1998). An introduction to special education, Bellco Publishers.

Omiegbe, O. (2001). Superstitious beliefs associated with the handicapped in Africa-African traditional religion: A book of selected readings (A. Orubu (ed.)).

Organization, W. H. (2001). International Classification of Functioning, Disability and Health. WHO.

Oyebode, A. (2009). Secret oath-taking is illegal. Nigerian Vanguard, 36.

Ozoji, E. D. (1991). Psychology of attitudes towards the disabled: The Nigerian perspective Department of Special Education.

Perrin, J. M. (2002). Health Services Research for Children with Disabilities. Milbank Quarterly. 80(2), 303-324.

Prince, M., Patel, V., Saxena, S., Maj, M., Maselko, J., Phillips, M. R., \& Rahman, A. (2007). No

Sawyer, S., Drew, S., Yeo, M. S., \& Britto, M. T. (2007). Adolescents with a chronic condition: Challenges Living, Challenges Treating. Lancet, 369, 1481--1489.

Susan, W. (1996). The Rejected Body: Feminist Philosophical Reflections on Disability, Routledge, New York. 46.

Wendy, T. (2012). Psychological and Social Aspects of Disability. Disabled World Journal, $12-14$.

WHO. (2004). Promoting Mental Health; Concepts Emerging Evidence and Practice Summary Report.

Yeomans, J., \& Citrome. (2005). Do Guidelines for Severe Mental Illness Promote Physical Health and Well-Being? Psychopharmacol. 19, 102-109. 